Née à Rimouski, Lucienne Lacasse-Lovsted vit à Toronto. Elle est membre de la Société des écrivains de Toronto et de l'Association des auteures et auteurs de l'Ontario français. Elle a publié un récit autobiographique, Une enfance rimouskoise (Rimouski: Éditeq, 1988) et quatre romans jeunesse, Une fille super (Saint-Boniface: Éditions des Plaines, 1994), Une grande sportive (Éditions des Plaines, 1995), Trop de kilos? (Vanier: CFORP, 1997), Narcos, machos, motos (Éditions des Plaines, 1999). Un recueil de poèmes, Puisque les fleuves nous conduisent, paraît en 1999 aux Éditions du GREF, à Toronto.

\title{
$\$$
}

\section{AUBE D'ÉTERNITÉ}

\section{Phase I :}

\section{L'Innommable}

I nsidieux

tu cherches le nid douillet

où te nicher

proliférer

Trois nodules à peine visibles prennent la grosseur d'une fève d'une fève de Lima

d'une gourgane

Monstre

tapi dans l'enchevêtrement des viscères pendant que le dépistage se poursuit, tout ce temps, gourgane

tu prolifères, triples, deviens boursouflure violacée oeil malin

tourné vers l'intérieur

Menace diffuse, anonyme, que je prétends ignorer dont je me cache la présence pour cacher ma frayeur bloquer ton avance ignorance cultivée

Toi Goliath.

Moi David.

Mon caillou :

mon rire.

Rire qui exorcise la peur

oublie ton intrusion

rassure les miens

Rire aujourd'hui

pour rire au dernier jour

pour un exit en beauté

Première escarmouche, tu me découronnes

Frustration

tête standard

banale à faire craquer le miroir

qui reflète des frisettes

rêches au toucher

sans l'éclat argenté

de ma chevelure déracinée

Me démoraliser

me montrer qui est maître

un jeu? 
Mais l'arsenal des artifices

est bien équipé...

les perruques,

c'est pas pour les chiens

et les cheveux repoussent

Cratère,

tu suintes une poisse fétide

étends tes ramifications

entraves le mouvement

mais à part ça

Madame la marquise

tout va très bien, tout va très bien!

Première victoire

le cocktail chimique

te fait baisser pavillon

Sans relâcher le terrain gagné

tu reviens mener le bal

Enlacements furieux

étreintes brutales de la douleur

tango lascif,

danse macabre

qui me coupe le souffle

Sur une plage de chair rose tu as rampé en silence avancé une pince exploratrice Crabe, tu chemines en silence

Je me stabilise essaie de deviner tes intentions tu prends plaisir à me terrifier

Lutter, défier

s'agripper... ou laisser aller

voilà la question
Panique

amplifiée par les ténèbres de la nuit

par les battements d'un coeur

qui se raconte ses peurs

d'un corps dont je questionne

le moindre gargouillement

Puis l'aube rosit le jour

sa luminosité réveille l'espoir.

N'était-ce qu'un cauchemar?

J'interroge le miroir

la silhouette ne paie pas de mine :

jambes

éléphantesques, bosselées, chancelantes, pieds

qui n'enjambent plus l'obstacle

eux qui hier encore m'emmenaient partout

marcheuse intrépide que j'étais

ventre

qui deux fois a porté la vie,

difforme, énorme

bras

qui font craquer les manches du peignoir

poignet

enflé à ne plus permettre les bracelets

visage

frais, rosé

qui défie ta présence par le sourire.

Et mon rire!

Panique (une fois de plus)

dissipée par la clarté qui pointe

ton ombre chassée par sa lumière

Inondée par l'espoir

renée avec le jour tout neuf

encore une fois

je jure de regarder la mort en face 
de réussir ma sortie de ce monde moi, non consultée à l'entrée

Quelles seront tes batteries pour l'assaut final?

Comment viendras-tu à moi patte de velours ou griffes sorties?

D'ici là tu prends plaisir par une boursouflure ici une bosse là à me rappeler ta présence à m'épeler le nom qui met fin â la charade.

\section{Phase II :}

\section{Ton nom est Cancer}

\section{CANCER}

Ton nom

épelé, collé à la réalité, sème en moi la stupeur

la panique

la finalité

Cela n'arrive qu'aux autres...

Statistiques à l'appui complices serviles qui tronquent les résultats

Signatures de constats de décès incontestables irréfutables : mort par arrêt cardiaque meurt-on d'autre chose?
J'ai anesthésié mon entendement nié, refusé Bravoure d'autruche à laquelle il faut mettre fin pour vivre dans la vérité, la vérité qui me rendra libre

Pour te combattre il faut te reconnaître te nommer, parer tes coups réalité du monde nouveau que tu es

J'absorbe un vocabulaire étranger. j'apprends les éléments du combat tout instinct de vivre réveillé.

Je contracte une alliance avec ceux qui, sur la brèche, t'observent, te déjouent, te maîtrisent de plus en plus, m'offrent un futur

\section{Futur incertain}

dont la précarité fait la force.

Débusquée, ton emprise diminuée, tu n'es plus qu'une maladie en perte de terrain

Toi qui mets l'odieux de ton oeuvre au compte des effets secondaires des agents de ton annihilation

Tu camoufles tes victoires crées une fausse sécurité falsifies les tables de morbidité pour poursuivre dans l'ombre tes victimes encore trop nombreuses 
Les cocktails chimiques n'ont plus d'effet Je crains ma perte novice que je suis à ton jeu cruel

Une arme plus puissante m'est offerte qui compte des cures miraculeuses Cobalt 60

Insecte anonyme

sous la loupe d'un entomologiste tel je suis

sous l'oeil qui cible la longueur, la largeur, la profondeur de la tumeur qui a crevé

Dix fois quatre-vingt-dix secondes

le rayon miraculeux brûle, réduit, assèche, redonne l'espoir

\section{Phase III :}

\section{Amnistie}

La médecine fait des miracles, il faut y croire... coopérer

La vie continue je me fais à ta présence à profiter des sursis que la Médecine t'arrache malgré tes tracasseries je chemine avec toi

Comme le marin qui cherche d'où souffle le vent je hume l'atmosphère qui est tienne je déchiffre tes moindres manifestations je t'interroge
Que fais-tu dans ma vie?

Pourquoi m'as-tu choisie?

Que m'offres-tu?

Le sens de la vie

la mort

la résurrection glorieuse

ou l'éternité du cosmos

molécule infime

je n'appartiendrais qu'à l'univers matériel celui du firmament où courent les nuages au rythme que les vagues

ont inscrit en moi ?

Ma vie sur un écran mon passé où défilent

les bons moments

les pénibles

les insignifiants

Présent

plein de tendresses

d'amitiés

Futur

aux lumières d'aube

La douleur m'a de nouveau visité cette nuit douleur avec 'd' minuscule

en basse région

J'avais sublimé

cette douleur annoncée

que $j$ 'attendais comme un noble creuset

où fondraient les scories

s'affinerait mon esprit

enfin libéré 
vulgaire colique

boyaux tordus

malaise sans gloire

annonciatrice du nouveau site choisi

De nouveau tu me nargues

tu sors tes griffes

Crabe,

nom que t'a donné Hippocrate

pour t'identifier

Moi qui te nomme Chat

Chat aux griffes camouflées

par le velours de ta fourrure

graffitant ma main

trahie par ton ronronnement

Chat aux éraflures sanglantes

qui ne m'empêche plus de sentir

les nodules gonflées

les pitons noirâtres

l' oedème qui m'alourdit
La douleur

aujourd'hui régit mes activités

vole les heures destinées à l'écriture...

Tyran qui me soumet à ton caprice

Morphine

Relâche inespérée

Brume dissipée

bouée à laquelle je me raccroche

Acheminement vers la dépendance

Renonciation aux efforts physiques

Acceptation des bons soins offerts

Reddition inconditionnelle congé des thérapies exigeantes Libération des soucis matériels en faveur de l'esprit recherche de la seule issue importante le sens de l'éternité vers laquelle tu me conduis 